\title{
Pilot study of vibration stimulation on neurological rehabilitation
}

\author{
Jianfeng Sui ${ }^{\mathrm{a}}$, Pete Shull ${ }^{\mathrm{b}}$ and Linhong $\mathrm{Ji}^{\mathrm{a}}{ }^{\mathrm{*}}$ \\ ${ }^{a}$ Department of Mechanical Engineering, Tsinghua University, Beijing 100084, China \\ ${ }^{b}$ Department of Mechanical Engineering, Stanford University, Stanford 94305, America
}

\begin{abstract}
Robot-assisted therapy has been proved effective for dyskinesia, and has many unique advantages compared with traditional treatment, such as repeatability, accuracy, objectivity. But some studies show that the effect of the robot-assisted rehabilitation for improving patients' activities of daily life (ADLs) is not obvious. This study introduces a novel auxiliary method-vibration stimulation combined with robots which may improve patients' ADLs. In controlled trials, two kinds of feedback-vibration and visual feedback are applied to prompt subjects for rehabilitation, and electromyographic signals (EMGs) and motion parameters are recorded in real time. Experimental results show that subjects' EMGs using vibration feedback are similar to healthy people, and characteristics of motion are closer to the theoretical value compared with control group. Vibration stimulation may serve as a kind of efficient auxiliary means to improve the efficiency of neurological rehabilitation.
\end{abstract}

Keywords: Vibration stimulation, neurological rehabilitation, neuroplasticity, rehabilitation robot

\section{Introduction}

Incidence and disability caused by neurological damage is increasing worldwide bringing serious burdens to society and patients' families [1,2], and finding reasonable treatments to solve this problem is difficult. The introduction of robots into this area is a major breakthrough [3]. In comparison with conventional therapy the robot-assisted rehabilitation has many advantages, such as manpower-saving, good repeatability, high accuracy, and great objectivity. The purpose of rehabilitation is to enable patients to complete tasks accurately and improve their activities of daily life (ADLs) [4].

Neuralplasticity provides a theoretical basis for neurological rehabilitation [5]. Robot-assisted rehabilitation aims not only to improve motor ability of limb muscles, but more importantly, to maximize the recovery of movement and control ability of the nervous system [6,7]. These robot-assisted therapies should be based on neuroscience, which is one the most efficient and reasonable means of rehabilitation [8]. Some studies have shown that robot-assisted rehabilitation does not improve patients' ADLs, because task-oriented contents and methods are effective for dyskinesia and neglect nervous system [9]. In order to improve the rehabilitation, enjoyment, and patients' active

${ }^{*}$ Corresponding author: Linhong Ji, Department of Mechanical Engineering, Tsinghua University, Beijing 100084, China. Tel./Fax: +86010 62785591; E-mail: jilh@tsinghua.edu.cn. 
participation, some innovative methods have been introduced into the rehabilitation system. Using visual feedback, patients can concentrate on their actions and proactively adjust trajectories to match their target. Combined with virtual reality technology to simulate actual situation, the training becomes more vivid and concrete. Adding is video games can increase patients' confidence and active participation in rehabilitation [10].

Over the last few years, the study of mechanical vibration has been widespread concern, and many relevant studies prove its validity [11-16]. Vibrational frequency between 20 and $50 \mathrm{~Hz}$ applied to the whole-body is primarily used to ease muscle tension and fatigue during sports practice, improve balance, coordination and muscular endurance, and prevent osteoporosis $[11,12]$. When vibration frequency between 80 and $120 \mathrm{~Hz}$ is utilized, it is possible to effectively stimulate Ia nerve fibers, resulting in wave I to stimulate the sensorium motor cortex region, to improve the effectiveness of rehabilitation and help neural remodeling [13,14]. Vibration frequencies between 200 and $300 \mathrm{~Hz}$ (especially $250 \mathrm{~Hz}$ ) can effectively stimulate proprioception, especially as a tactile prompt $[15,16]$.

This paper illustrates the effectiveness of vibration stimulation compared with visual feedback applied to robot-assisted therapy, which may serve as an auxiliary means to improve the efficiency of neural rehabilitation.

\section{Principles and systems}

\subsection{Vibrotactile principle}

Methods to obtain external information are mainly conducted by the human sensory system, of which haptic modality is one of the most important aspects. Mechanical vibration stimulating haptic sensation to obtain information is one focus of research in recent years [17,18]. Haptic modality is a sense caused by a weak mechanical stimulation exciting skin shallow receptors. It must be noted that the mechanical aspect of touch is transduced in the periphery by four different types of mechanical receptors: Merkel, Ruffini endings, Meissner's and Pacinian corpuscles. Each one has a particular morphology, sensitive range, receptive field size, and response pattern over time. All four types of receptors are commonly involved to varying degrees in the haptic experience of contact with everyday objects and surfaces. These receptors are densely distributed in joints, and so the vibrational touch in joints is more sensitive. Verrillo found that the most sensitive vibration frequency range is $200-300$ $\mathrm{Hz}$, especially $250 \mathrm{~Hz}$. Tan et al. [17] found that effective vibration could reduce reaction time compared to visual feedback through experiments with vibrators on the back of 10 college students (5 male,5 female) (frequency: 250-300 Hz, amplitude: $27 \mathrm{db}$ ) in 2003. Wall et al. [18] found that vibrational touch (frequency: $250 \mathrm{~Hz}$ ) could effectively help participants adjust posture and improve gait. During the experiment nine subjects ( 5 male, 4 female) participated and their body posture and plantar pressure were recorded in 2012.

\subsection{Rehabilitation training systems}

\subsubsection{Multi-posture upper-extremity training device (MPUTD)}

The MPUTD has three degrees of freedom, a linear motor to make the motion plane rotate to extends the training space into three-dimensional space, and two rotary motors that coordinate to accomplish shoulder and elbow movement [19]. The device has two sets of monitors, one that is in front of patients and the other that is in the movement plane (following the rotation of the plane) so 
subjects can observe their real-time trajectory in the proper perspective, as shown in Figure 1. The MPUTD can provide three training modes: passive, active-constrained, and damping mode. Subjects can choose the proper training models depending on their stage of recovery. The device can provide two training strategies: linear motion and circular motion. The length of the straight line and the size of the circle radius can adjust automatically, accounting for patients' individual differences.

\subsubsection{Vibration perception system}

The vibration perception system is composed of an angle sensor, two C2 tactors, and two plastic plates. The $\mathrm{C} 2$ tactor as shown in Figure 2 is able to provide mechanical vibration of different frequency and amplitude. The frequency of $250 \mathrm{~Hz}$ was found most sensitive to experimenters by previous experiments [20]. Depending on patients' capacity, appropriate amplitudes are selected. Two tactors are respectively attached to the internal and external sides of the patients' forearm. An Angle range is set in the program in advance; when the elbow angle is larger than the maximum threshold, the vibrator inside sends signals to remind patients do adduction movements. When the angle is less than minimum threshold, the vibrator outside sends signals to remind patients to do outreach movement.

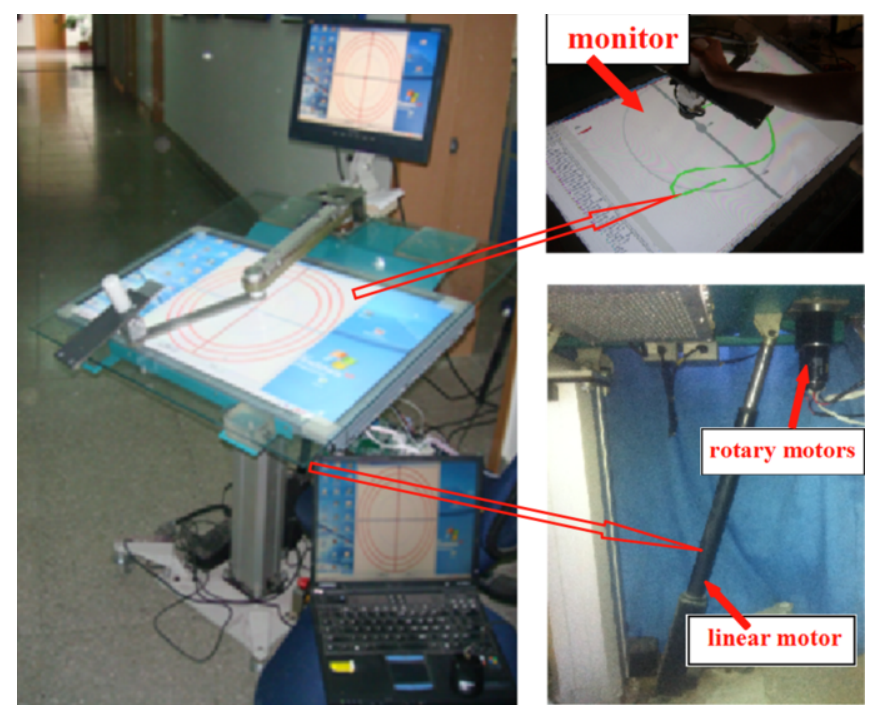

Fig. 1. Multi-posture upper-extremity training device (MPUTD).

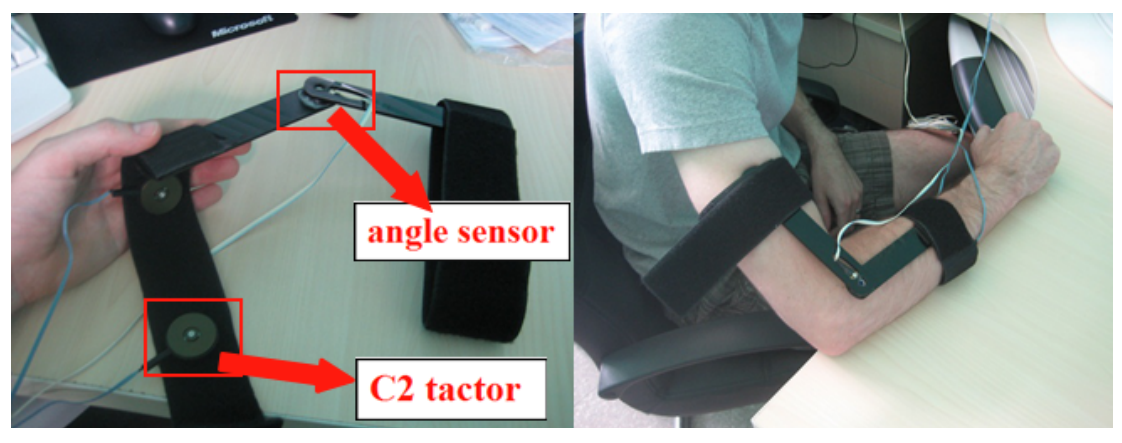

Fig. 2. Vibration perception system. 


\section{Experiments and the results}

\subsection{Subjects}

The active-constrained mode of the MPUTD was used throughout the experiment. Subjects had some athletic ability, but there were still some defects in their neural control, so they could not accurately control their movements. Subjects who were in the Brunnstrom [21] 3-4 stage met the experimental conditions. Five patients (three women and two men) were selected as subjects, aged between 40 and 60 years old, with no defects of understanding, 3-6 months after stroke and in good rehabilitation conditions.

\subsection{Experimental Protocol}

The prompt effect of vibration stimulation was tested compared with visual feedback during controlled trials as shown in Figure 3. Before experiments, subjects were informed how to use the MPUTD, vibration perception system and relevant precautions. Before the formal experiments, subjects practiced using the MPUTD for some time to become familiarized with the device. Electrodes were affixed to six muscles of the upper extremities: the brachioradialis, biceps, triceps, anterior deltoid, lateral deltoid and posterior deltoid to collect electromyographic signals (EMGs). Surface EMG signals (band-pass: 0-200 Hz, sampling rate $1500 \mathrm{~Hz}$ ) were recorded using surface $\mathrm{Ag}-\mathrm{AgCl} 2.5 \mathrm{~cm}$ square pre-gelled electrodes placed over these six muscle bellies on lightly abraded skin. Under active-constrained mode, subjects did straight reciprocating motion with 5 second cycles. In the contrast experiment, using visual feedback, subjects could observe their real-time trajectory to correct their action, and the training time was 10 minutes. After resting for half an hour (first, to prevent impact of fatigue on the result of patients' EMGs; second, to release the memory of the last training to reduce the interference on the next one), subjects wore the vibration perception system with the screen off, also for 10 minutes. EMGs and motion characteristics of subjects were recorded during these two processes.

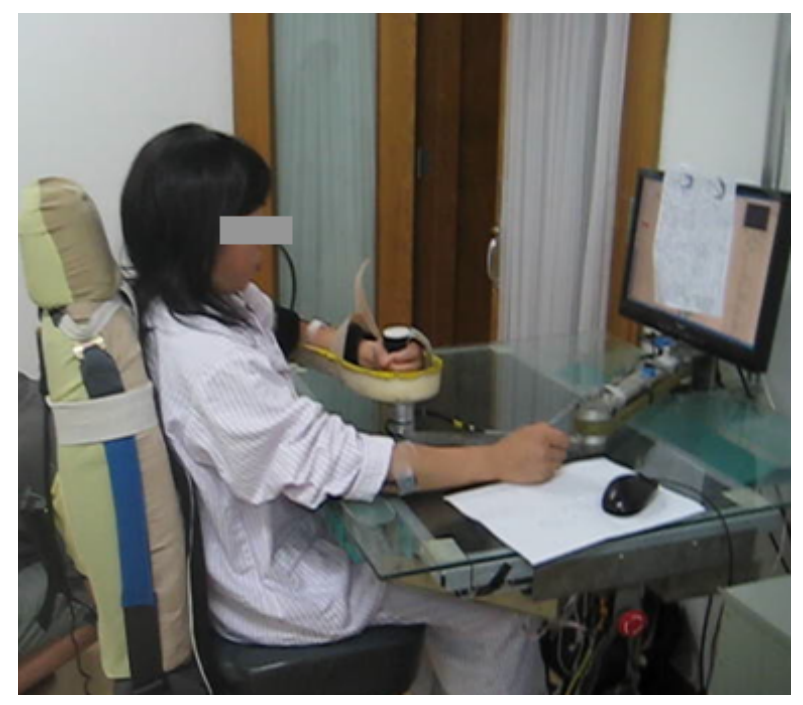

Fig. 3. Experimental process of subject 1. 

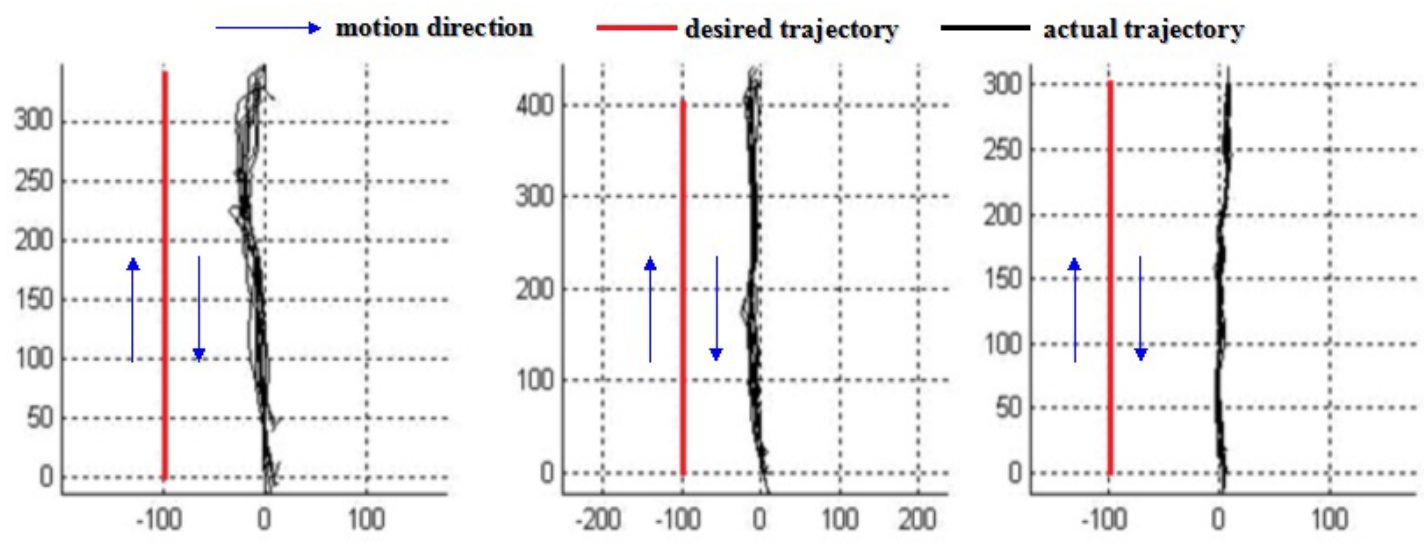

Fig. 4. The trajectories of subjects.

\subsection{Results}

The length of the straight reciprocating motion was determined according to the range of each subject's movement. Blue arrows represent the motion direction of the subject, red solid lines represent the theory trajectory, and black solid lines represent the actual trajectory as shown in Figure 4. The angle range of the elbow joint was a fixed in the test, and arm length of each subject was different. Accordingly the trajectory lengths of the upper-limb are different as the red and black solid lines shown in the figure.

\subsubsection{EMGs}

EMGs of these six muscles were processed through a 4th order Butterworth low-pass filter $(30 \mathrm{~Hz})$ to remove artificial clutter noise such as power line interference, and then through a full-wave rectifier, and finally through a 1st order Butterworth low-pass filter ( $6 \mathrm{~Hz}$ for cut-off frequency) [22], as shown in Figure 5. This showed that in motion processes, the training intensity of muscles and nervous system using vibration stimulation was greater, and the effect was more pronounced compared with visual feedback. Muscle contribution percentage was calculated to analyze the correlation of muscle and movement, according to the following equation:

$$
W_{i}=\frac{S_{i}}{\sum_{i=1}^{n} S_{i}} \%
$$




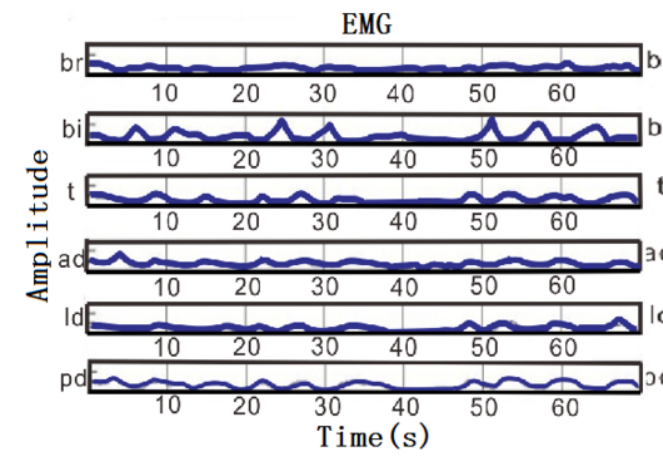

(a)

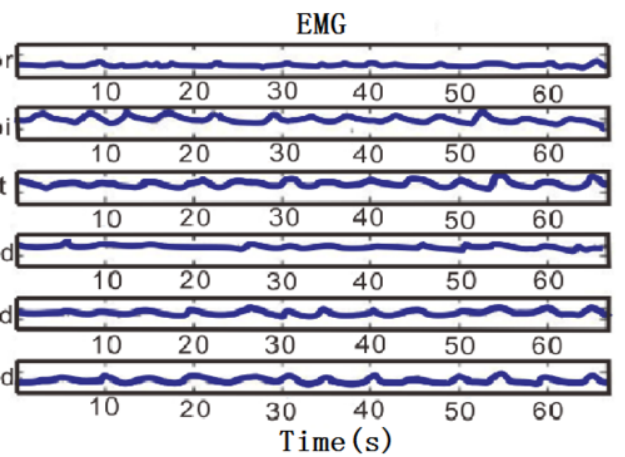

(b)

Fig. 5. (a) EMG average of patients using visual feedback. (b) EMG average of patients using vibration stimulation. br-brachioradialis, bi--biceps, t--triceps, ad--anterior deltoid, ld--lateral deltoid, pd--posterior deltoid.

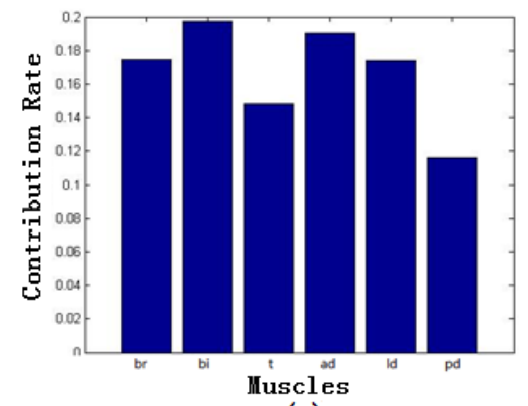

(a)

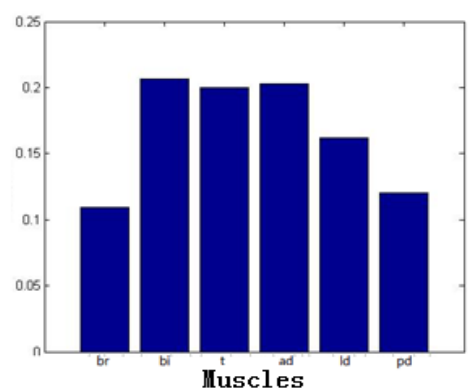

(b)

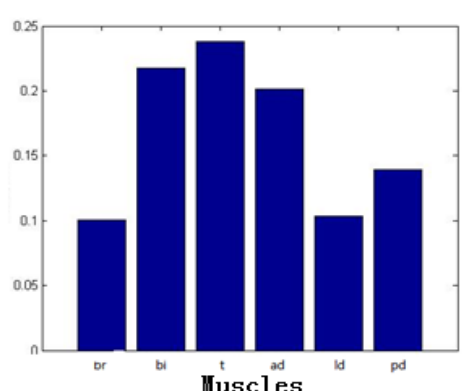

(c)

Fig. 6. (a) Contribution percentage of each muscle to the movement using visual feedback. (b) Contribution percentage using vibration stimulation. (c) Contribution percentage of normal person's each muscle.

Where $W_{i}$ represents contribution percentage of muscle i during all these six muscles in this movement, and $S_{i}$ represents amplitude of muscle $\mathrm{i}$ in the time domain. The histogram which represented contribution percentages of each muscle to the straight reciprocating motion was shown in Figure 6. Contribution percentages of the same muscle in the same action was also different using these two feedbacks, and subjects using vibration stimulation were closer to normal person in the comparison group.

\subsubsection{Movement features}

Elbow joint movement angles of subject's change over time were measured and recorded by angle sensor of the vibration perception system, and 80 seconds of 10 minutes of data (Subject 2--S2) were as shown in Figure 7. The cycle of straight reciprocating motion was $5 \mathrm{~S}$ (frequency was $0.2 \mathrm{~Hz}$ as shown by blue line in figure). The phase of actual trajectory lagged behind the desired trajectory using visual feedback, while the phase of actual trajectory matched better with desired trajectory using vibration stimulation. The data were analyzed in the frequency domain according to the following equation: 


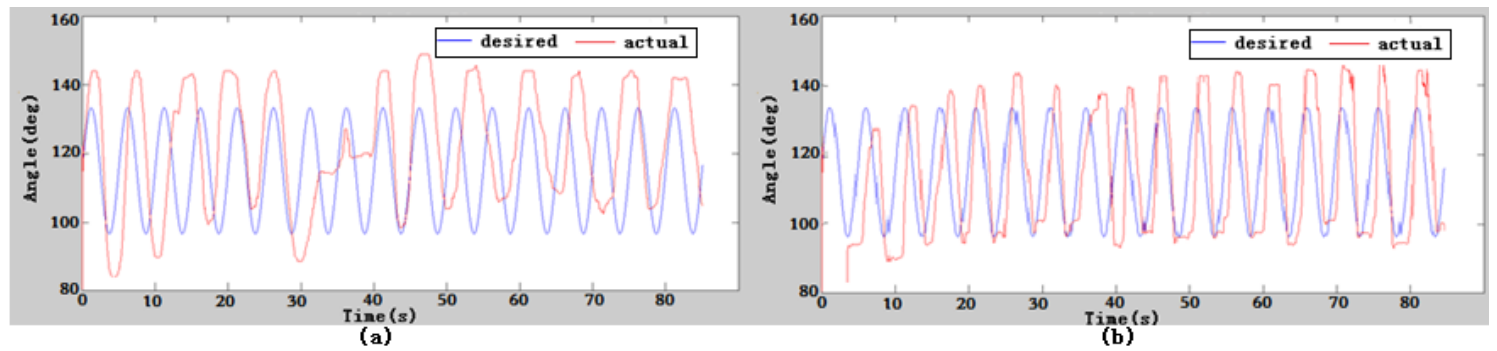

Fig. 7. (a) Movement features using visual feedback (S2). (b) Movement features using vibration stimulation (S2).

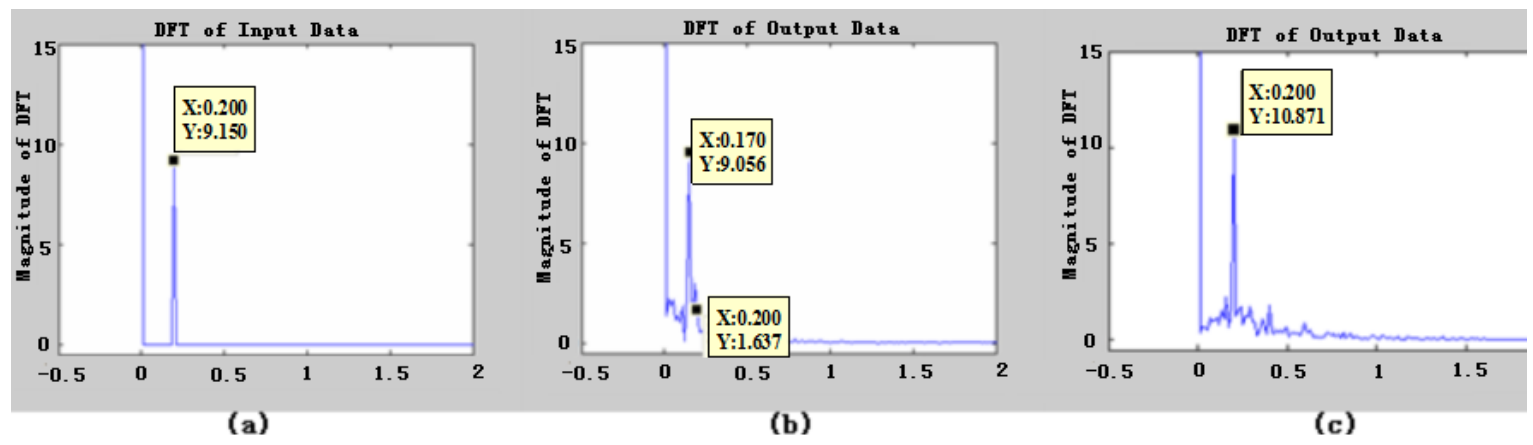

Fig. 8. (a) Desired movement frequency $0.2 \mathrm{~Hz}$. (b) Movement features in frequency domain using visual feedback (S2). (c) Movement features in frequency domain using vibration stimulation (S2).

$$
P(f)=\frac{1}{N}\left|\sum_{n=1}^{N} w(n) x(n) e^{-j \omega n}\right|^{2}
$$

$P(f)$ represents signals in frequency domain, $w(n)$ is window function (Blackman), $x(n)$ represents discrete signal in time domain. The results were shown in Figure 8. The main frequencies of five subjects using visual feedback were $0.14-0.18 \mathrm{~Hz}(\mathrm{~S} 1: 0.148, \mathrm{~S} 2: 0.170, \mathrm{~S} 3: 0.179, \mathrm{~S} 4: 0.178$, $\mathrm{S} 5: 0.177)$, the average was $0.171( \pm 0.013) \mathrm{Hz}$, while the main frequency using vibration stimulation were $0.18-0.20 \mathrm{~Hz}(\mathrm{~S} 1: 0.192, \mathrm{~S} 2: 0.200, \mathrm{~S} 3: 0.185, \mathrm{~S} 4: 0.194, \mathrm{~S} 5: 0.187$, the average was $0.192( \pm 0.006)$ $\mathrm{Hz}$, almost consistent with the frequency of the desired movement.

\section{Conclusion and discussion}

In our study, multi-posture upper-extremity training device (MPUTD) combined with the vibration perception system is applied to the neurological rehabilitation. By comparing EMGs and motion parameters of subjects using visual feedback and vibration stimulation these two feedbacks, vibration stimulation has distinct advantages in prompt. Robot-assisted rehabilitation with vibration stimulation may improve the efficiency of neurological rehabilitation.

Upper-extremity robot-assisted rehabilitation therapy combined with vibration stimulation has good performance during neurological rehabilitation process. The experiment results show that the feedback 


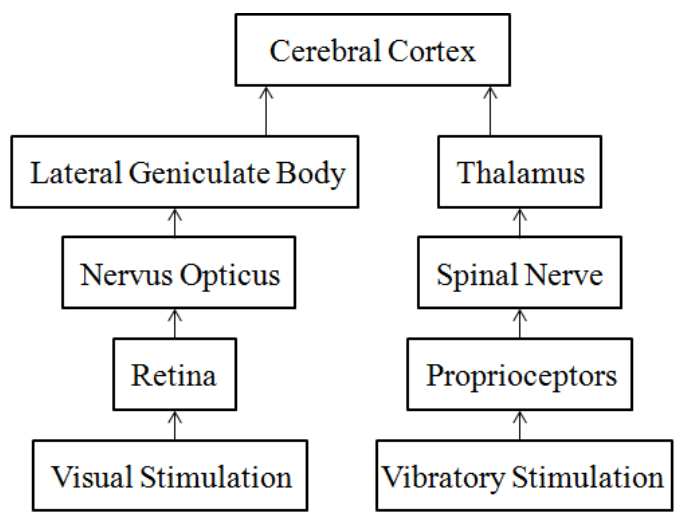

Fig. 9. Signaling pathways of two feedback.

time of vibration stimulation is shorter than visual stimulation consistent with the results of Tan et al. and Wall et al.'s research [17,18], signaling pathways as shown in Figure 9. This result may occur due to the time necessary to convert image information into electrical signals in visual feedback, while there is no signal transformation in vibration stimulation.

Haptic modality is an important way to obtain outside information for humans with four kinds of proprioceptive receptors in the skin which can accept different frequencies of vibration: Merkel, Ruffini endings, Meissner's and Pacinian corpuscles as mentioned earlier. Haptic sensitivity by vibration stimulation depends on many factors such as vibration position, gender, age, temperature, vibration frequency and time. Barghout [23] found these four proprioceptive receptors are widely distributed around joints, and the vibration around joints is sensitive, therefore, the vibration positions in the experiment are generally around joints.

The lower-extremity rehabilitation device with the vibration perception designed by Tsinghua University can simulate normal gait, and we attempt to apply this pattern to lower-extremity rehabilitation process. Through experiments, the effect of lower-extremity rehabilitation is worthy of continued study, but comparison experiments have been conducted. Further experiments need to be conducted to prove the efficacy of this pattern in neurological rehabilitation process of lower extremities, and several different methods of feedback (e.g. auditory feedback) should be compared to this auxiliary method of rehabilitation engineering.

\section{References}

[1] T. Truelsen, B. Piechowski-Jowiak, R. Bonita, C. Mathers, J. Bogousslavsky and G. Boysen, Stroke incidence and prevalence in Eurpoe: A review of avaikable data, Eur. J. Neurol. 13 (2006), 581-598.

[2] C. Gowland, Recovery of motor function following stroke: Predicting disability in stroke: A critical review of the literature, Age Ageing 25 (1996), 479-489.

[3] M. Stefano, A. Mario and R. Giulio, Upper-limb robot-assisted therapy in rehabilitation of acute stroke patients: Focused review an results of new randomized controlled trial, Journal of Rehabilitation Research \& Development $\mathbf{4 8}$ (2011), 355-366.

[4] C. Butefisch, H. Hummelsheim, P. Denzler and H. Mauritz, Repetitive training of isolated movements improves the outcome of motor rehabilitation of the centrally paretic hand, J. Neurol. Sci. 130 (1995), 59-68.

[5] M. Elior, M. Zeev, N. Martin, H. Nurith and S. Isabella, Spinal decompression sickness presenting as partial brown-sequard syndrome and treated with robotic-assisted body-weight support treadmill training, J. Rehabil. Med. 41 (2009), 88-89. 
[6] M. Wirz, D.H. Zemon, R. Rupp, A. Scheel, G. Colombo and V. Dietz, Effectiveness of automated locomotor training in patients with chronic incomplete spinal cord injury: A multicenter trial, Arch. Phys. Med. Rehabil. 86 (2005), 672-680.

[7] W. Larry, A. Lewis and R. Andreas, Exercise-mediated locomotor recovery and lower-limb neuroplasticity after stroke, Journal of Rehabilitation Research \& Development 45 (2008), 205-220.

[8] I. Yamamoto, N. Inagawa, M. Matsui, K. Hachisuka, F. Wada and A. Hachisuka, Research and development of compact wrist rehabilitation robot system, Bio-Medical Materials and Engineering 24 (2014), 123-128.

[9] T. Candace, P. Beth, E. Nicholas and W. Patricia, Efficacy of rehabilitation robotics for walking training in neurological disorders: A review, Journal of Rehabilitation Research \& Development 48 (20114), 387-416.

[10] M. Ana, A. Hendrik and P.A. Jules, Pilot study to test effectiveness of video game on reaching performance in stroke, Journal of Rehabilitation Research \& Development 48 (20114), 431-444.

[11] V.B. Issurin, D.G. Liebermann and G. Tenenbaum, Effect of vibratory stimulation training on maximal force and flexibility, Journal of Sports Sciences 12 (1994), 561-566.

[12] G.F. Dakota, The influence of whole body vibration on jumping performance, Biology of Sport 15 (1998), 157.

[13] J.D. Rollnik, S. Siggelkow and M. Schubert, Muscle vibration and prefrontal repetitive transcranial magnetic stimulation, Muscle \& Nerve 24 (2001), 112-115.

[14] K. Rosenkranz and J.C. Rothwell, Differential effect of muscle vibration on intracortical inhibitory circuits in humans, The Journal of Physiology 551 (2003), 649-660.

[15] P.B. Shull, K.L. Lurie and M.R. Cutkosky, Training multi-parameter gaits to reduce the knee adduction moment with data-driven models and haptic feedback, Journal of Biomechanics 44 (2011), 1605-1609.

[16] C. Wall and E. Kentala, Effect of displacement, velocity, and combined vibrotactile tilt feedback on postural control of vestibulopathic subjects, Journal of Vestibular Research 20 (2010), 61-69.

[17] H.Z. Tan, R. Gray and J.J. Young, A haptic back display for attentional and directional cueing, Haptics-e 3 (2003), $1-20$.

[18] C. Wall, D. Wrisley and L. Oddsson, Vibrotactile feedback of mediolateral trunk tilt or foot pressure increases locomotor performance in healthy older adults-a pilot study, 34th Annual International Conference of the IEEE Eng. Med. Biol. Soc., 2012, 6145-6148.

[19] C. Li, E. Liu and L. Ji, Mechanical structure improvement of an upper limb rehabilitation robot based on an equivalent kinematic model, Journal of Mechanics in Medicine and Biology 6 (2013), 1-12.

[20] R. Verrillo, Age related changes in the sensitivity to vibration, The Journal of Gerontology 35 (1980), 185-193.

[21] S. Brunnström, Movement therapy in hemiplegia: A neurophysiologic approach, J. Gerontol. 27 (1972), 290.

[22] F. Wang and Z. Ji, Application of the dual-tree complex wavelet transform in biomedical signal denoising, Bio-Medical Materials and Engineering 24 (2014), 109-115.

[23] A. Barghout, J. Cha, A.E. Saddik, J. Kammerl and E. Steinbach, Spatial resolution of vibrotactile perception on the human forearm when exploiting funneling illusion, IEEE International Workshop IEEE, 2009, 19-23. 